\title{
Photo-Induced Polarity Change of Photosensitive Polyimide with $o$-Nitrobenzyl Ester Side Chain
}

\author{
Yoshihito Ishida, Yuya Kawabe and Atsushi Kameyama* \\ Department of Chemistry, Kanagawa University, \\ 23-709, 3-27-1 Rokkakubashi, Kangagawa-ku Yokohama 221-8686, Japan \\ kameya01@kanagawa-u.ac.jp
}

\begin{abstract}
Photochemical reaction of the pendant $o$-nitrobenzyl ester of the polyimide films was investigated in details by FT-IR and UV-vis absorption spectra to confirm the generation of the corresponding carboxylic acid group. The conversions of the pendant $o$-nitrobenzyl ester increased with irradiation time and reached to $38-46 \%$ under $60 \mathrm{~min}$ irradiation. Water contact angle of PSPI 1-4 films decreased from $75^{\circ}$ to below $55^{\circ}$ after UV irradiation for 60 min followed by rinse treatment with toluene, in which $o$-nitroso benzaldehyde as a side product of the photochemical reaction was removed, due to the generation of carboxylic acid group. It was found that photochemical reaction of the pendant $o$-nitrobenzyl ester in the polyimide film having high $T_{\mathrm{g}} \mathrm{s}$ proceeded in middle conversion to induce water contact angle change as $20^{\circ}$.
\end{abstract}

Keysords: Polyimide, Photo Sensitive Polymer, o-Nitrobenzyl group, Polarity Change

\section{Introduction}

Polyimide (PI) is one of the most common super-engineering plastics that have been widely used in microelectronics due to their excellent thermal, mechanical and reasonable dielectric properties. For micro-fabrication of polyimide, photosensitive polyimide (PSPI) is one of the best materials because it can simplify the micro-fabrication process compared to the conventional system using additional photoresist layer [1-5]. Most of research in PSPI employed photochemical reactions that can induce large solubility change. Photo-irradiation of positive-type PSPI resulted in the increased solubility to a development solution, while that of negative-type PSPI form insoluble crosslinked polymer.

On the other hand, there is just few attempts that focused on the photo-induced polarity and wettability change of the PSPI. Photo-induced polarity and wettability change is attracting increasing attention in the micro-circuit fabrication and printed electronics. Because a surface have micro-pattern with different wettability can prevent the defect of wetting of metal-containing ink. To accomplish the large contrast of wettability between before and after photo irradiation, generation of polar functional groups such as carboxylic acids from esters with lower polarity by photo-irradiation is one of the considerable strategy.

$o$-nitrobenzyl ester and ether group are known as conventional photo-deprotection group for polar carboxylic acid and phenol group. Ryu et.al reported PSPI with side chain $o$-nitrobenzyl ester group for solubility change [6]. Photo irradiation cause deprotection of the $o$-nitrobenzyl group to generate polar benzoic acid group and resulted polymer is soluble in a developer. Several PSPI systems employing side chain $o$-nitrobenzyl ester and ether $[7,8]$ for solubility change were reported. However, it is difficult to introduce $o$-nitrobenzyl group to all side chain because $o$-nitrobenzyl group was introduced by post-polymerization reaction. In our previous report, we found chemoselective esterification of 5,5'-Methylenebis (2-aminobenzoic acid) (MBAA) with alkyl halide by using 1,8-diazabicylro[5,4,0]undec-7-ene (DBU) [9-11]. By using the functionalized diamine monomer, a series of polyimides with functional group at all 
side chain was successfully synthesized. The polyimides with side chain propargyl, vinyl ether and spiro otrho ester group was conducted to the photo-induced crosslink reaction based on the cationic polymerization of side chain in the presence of photo acid generator.

This paper describes the development of novel PSPI for large polarity and wettability change. Polyimides with $o$-nitrobenzyl ester group at all side group was synthesized by the condensation of diamine with $o$-nitrobenzyl ester and aromatic and aliphatic tetracarboxylic dianhydrides and subsequent chemical imidization. The diamine monomer was prepared by chemoselective reaction of carboxylic group containing diamine with $o$-nitrobenzyl chloride. The photochemical reaction of PSPIs and the influence of chemical structure and photo irradiation condition on the water contact angle was evaluated.

\section{Experimental Section}

\subsection{Materials}

5,5'-Methylenebis (2-aminobenzoic acid) (MBAA) was purchased from Wakayama Seika Kogyo Co. Ltd. o-nitrobenzyl chloride, 1,8diazabicylro[5,4,0]undec-7-ene (DBU), dimethyl sulfoxide (DMSO), 1-methyl-2-pyrroridione (NMP) and $N, N$-dimethyl acetoamide (DMAc), $N, N$-dimethylfromamide (NMF), pyridine, acetic anhydride were purchased from Wako Chemical Co. Ltd. and used after distillation over $\mathrm{CaH}_{2}$. Pyrromerit dianhydride (PDA), 4,4'(hexafluoroisopropyridene) diphtalic anhydride (6FDA), 3,3',4,4'-benzophenone tetracarboxylic dianhydrides (BTDA) 1,2,3,4-cycropentane tetracarboxylic dianhydrides (CPDA) was purchased from TCI and used after sublimation.

\subsection{Measurement}

The ${ }^{1} \mathrm{H}-\mathrm{NMR}$ spectra were obtained on a JEOL JNM-ECA-500 instrument at 500 and $100 \mathrm{MHz}$, respectively. DMSO- $d_{6}$ was used as a solvent with tetramethylsilane (TMS) as an internal standard. The infrared (IR) spectra were recorded on a JASCO FT/IR-4100 spectrometer. Numberand weight-average molecular weights $\left(M_{\mathrm{n}}\right.$ and $M_{\mathrm{w}}$ ) were determined by gel permeation chromatography (GPC) analysis with HLX8220 using RI detectors eluted with DMF at a flow rate of $1 \mathrm{~mL} / \mathrm{min}$ and calibrated by standard polystyrene samples. Thermogravimetric analysis (TGA) was performed on a Seiko
EXSTAR 6000 TG/DTA 6200 thermal analyzer at a heating rate of $10{ }^{\circ} \mathrm{C} / \mathrm{min}$ under a nitrogen flow. Differential scanning calorimetry (DSC) analyses were performed on a Seiko EXSTAR DSC 6200 at heating and cooling rate of $10{ }^{\circ} \mathrm{C} / \mathrm{min}$ under a nitrogen flow. UV-vis spectra were recorded on a Jasco V-670 spectrometer. Water contact angle of PSPI films was measured by Kyowa Interface Science Drop Master DM500.

\subsection{Synthesis of Monomer 1}

MBAA (1.00 g, $3.49 \mathrm{mmol})$ and DBU (1.17 g, $7.68 \mathrm{mmol})$ was dissolved in dry DMSO $(5 \mathrm{~mL})$. $o$-Nitrobenzyl chloride $(1.32 \mathrm{~g}, 7.68 \mathrm{mmol})$ was added dropwise at ambient temperature and stirred for $6 \mathrm{~h}$. The reaction mixture was precipitated into $400 \mathrm{~mL}$ of water. The yellow powder was collected and dried in vacuo to obtain crude product. The crude product was purified by alumina column chromatography with $\mathrm{CHCl}_{3} /$ hexane/ethyl acetate $(3: 4: 3, \mathrm{v} / \mathrm{v} / \mathrm{v})$ as an eluent and light yellow powder was obtained (yield: $0.697 \mathrm{~g}, \quad 35 \%) . \quad{ }^{1} \mathrm{H}-\mathrm{NMR} \quad(500 \mathrm{MHz}$, DMSO- $\left.d_{6}\right) \delta(\mathrm{ppm}): 8.09(\mathrm{~d}, J=1.7 \mathrm{~Hz}, 2 \mathrm{H}), 7.76$ (t, $J=6.3 \mathrm{~Hz}, 2 \mathrm{H}), 7.65(\mathrm{~d}, J=6.9 \mathrm{~Hz}, 2 \mathrm{H}), 7.60(\mathrm{t}$, $J=7.4 \mathrm{~Hz}, 2 \mathrm{H}), 7.55(\mathrm{~s}, 2 \mathrm{H}), 7.14(\mathrm{~d}, J=6.9 \mathrm{~Hz}$, $2 \mathrm{H}), 6.74(\mathrm{~d}, J=8.6 \mathrm{~Hz}, 2 \mathrm{H}), 6.58(\mathrm{~s}, 4 \mathrm{H}), 5.57(\mathrm{~s}$, $4 \mathrm{H}), 3.67(\mathrm{~s}, 2 \mathrm{H})$. FT-IR $\left(\mathrm{KBr}, \mathrm{cm}^{-1}\right): 3479\left(\mathrm{v}_{\mathrm{N}-\mathrm{H}}\right)$, $3373\left(v_{\mathrm{N}-\mathrm{H}}\right), 2850\left(v_{\mathrm{C}-\mathrm{H}}\right), 1689\left(v_{\mathrm{C}=\mathrm{O}}\right), 1522\left(\mathrm{v}_{\mathrm{asNO} 2}\right)$, $1340\left(v_{\mathrm{sNO} 2}\right)$.

\subsection{Typical Synthetic Procedure of PSPIs}

To a solution of $1(0.557 \mathrm{~g}, 1.00 \mathrm{mmol})$ in dry DMSO $(2.3 \mathrm{~mL})$, tetracarboxylic acid dianhydride $(1.00 \mathrm{mmol})$ was added and stirred at r.t. for $6 \mathrm{~h}$. Acetic anhydride $(0.73 \mathrm{~g}, 7.10 \mathrm{mmol})$ and pyridine (0.39 g, $4.88 \mathrm{mmol})$ was added to the reaction mixture and stirred at $80^{\circ} \mathrm{C}$ for $4 \mathrm{~h}$. The solution was precipitated into $\mathrm{MeOH}$ and the dried in vacuo to obtain pale orange powder. PSPI 1: $0.68 \mathrm{~g}$, $89 \%$. ${ }^{1} \mathrm{H}-\mathrm{NMR}\left(500 \mathrm{MHz}, \mathrm{DMSO}-d_{6}\right) \delta(\mathrm{ppm})$ : 8.20-7.47 (br, 16H), 5.79 (br, 4H), 3.36-3.32 (br, 2 H). FT-IR $\left(\mathrm{KBr}, \mathrm{cm}^{-1}\right): 1779\left(\mathrm{v}_{\mathrm{asC}=\mathrm{O}}\right), 1729\left(\mathrm{v}_{\mathrm{sC}=\mathrm{O}}\right)$, $1672\left(v_{\mathrm{C}=\mathrm{O}}\right), 1527\left(\mathrm{v}_{\mathrm{asNO} 2}\right), 1505\left(\mathrm{v}_{\mathrm{C}=\mathrm{C}}\right), 1257\left(\mathrm{v}_{\mathrm{C}-\mathrm{N}}\right)$. $M_{\mathrm{n}}=12,000, M_{\mathrm{w}} / M_{\mathrm{n}}=2.25$. PSPI 2: $0.86 \mathrm{~g}$, $86 \% . \quad{ }^{1} \mathrm{H}-\mathrm{NMR}\left(500 \mathrm{MHz}, \mathrm{DMSO}-d_{6}\right) \delta(\mathrm{ppm})$ : 8.10-7.49 (br, 20H), 5.47-5.44 (br, 4H), 4.30 (br, 4H). FT-IR $\left(\mathrm{KBr}, \mathrm{cm}^{-1}\right): 1786 \quad\left(\mathrm{v}_{\mathrm{asC}=\mathrm{O}}\right), 1728$ $\left(v_{\mathrm{SC}=0}\right), 1675\left(v_{\mathrm{C}=\mathrm{O}}\right), 1529\left(v_{\mathrm{NO} 2}\right), 1505\left(v_{\mathrm{C}=\mathrm{C}}\right), 1257$ $\left(v_{\mathrm{C}-\mathrm{N}}\right), 1297\left(v_{\mathrm{SC}-\mathrm{F}}\right) . \quad M_{\mathrm{n}}=8,500, M_{\mathrm{w}} / M_{\mathrm{n}}=2.21$. PSPI 3: 0.86 g, 98\%. ${ }^{1} \mathrm{H}-\mathrm{NMR}(500 \mathrm{MHz}$, DMSO- $\left.d_{6}\right) \delta(\mathrm{ppm}): 8.02-7.23(\mathrm{br}, 20 \mathrm{H}), 5.16(\mathrm{br}$, 4H), 4.05 (br, 2H). FT-IR $\left(\mathrm{KBr}, \mathrm{cm}^{-1}\right): 1780$ 
Table 1. Preparation of PSPIs ${ }^{\text {a) }}$ and Their Thermal Properties

\begin{tabular}{ccccccccc}
\hline Polyimide & Monomer & $\begin{array}{c}\text { Polymerization } \\
\text { Time }(\mathrm{h})\end{array}$ & $\begin{array}{c}\text { Yield } \\
(\%)\end{array}$ & $M_{\mathrm{n}}{ }^{\mathrm{b})}$ & $M_{\mathrm{w}} / M_{\mathrm{n}}{ }^{\mathrm{b})}$ & $\begin{array}{c}T_{\mathrm{g}}{ }^{\mathrm{c})} \\
\left({ }^{\circ} \mathrm{C}\right)\end{array}$ & $\begin{array}{c}T_{\mathrm{d} 5 \%}{ }^{\mathrm{d})} \\
\left({ }^{\circ} \mathrm{C}\right)\end{array}$ & $\begin{array}{c}T_{\mathrm{d} 10 \%}{ }^{\mathrm{d})} \\
\left({ }^{\circ} \mathrm{C}\right)\end{array}$ \\
\hline PSPI-1 & PDA & 6 & 89 & 12,000 & 2.25 & 174 & 293 & 313 \\
PSPI-2 & 6FDA & 6 & 86 & 8,500 & 2.21 & 173 & 304 & 353 \\
PSPI-3 & BTDA & 12 & 98 & 5,400 & 1.96 & 163 & 294 & 316 \\
PSPI-4 & PCDA & 6 & 50 & 1,700 & 1.29 & 122 & 268 & 289 \\
\hline
\end{tabular}

${ }^{a}$ the polymerization reaction was conducted with MBAA and tetracarboxylic dianhydride in dry DMSO at r.t. Chemical imidization was conducted at $80{ }^{\circ} \mathrm{C}$ for $4 \mathrm{~h}$ by acetic anhydride and pyridine. ${ }^{b}$ Measured by GPC with DMF as an eluent (PS standards). ${ }^{c}$ Measured by DSC under $\mathrm{N}_{2}$ flow at a heating rate of $10{ }^{\circ} \mathrm{C} / \mathrm{min}$. ${ }^{d}$ Measured by TGA under $\mathrm{N}_{2}$ flow at heating rate of $10^{\circ} \mathrm{C} / \mathrm{min}$.

$\left(v_{\mathrm{asC}=0}\right), 1719\left(v_{\mathrm{sC}=\mathrm{O}}\right), 1612\left(v_{\mathrm{C}=\mathrm{O}}\right), 1526 \quad\left(v_{\mathrm{asNO}}\right)$, $1506\left(v_{\mathrm{C}=\mathrm{C}}\right), 1264\left(v_{\mathrm{C}-\mathrm{N}}\right) . \quad M_{\mathrm{n}}=5400, M_{\mathrm{w}} / M_{\mathrm{n}}=$ 1.96. PSPI 4: $0.38 \mathrm{~g}, 50 \%$. ${ }^{1} \mathrm{H}-\mathrm{NMR}(500 \mathrm{MHz}$, DMSO- $\left.d_{6}\right) \delta(\mathrm{ppm}): 8.13-7.38(\mathrm{br}, 14 \mathrm{H}), 5.63-5.53$ (br, 4H), 4.25 (br, 2H), 2.13-2.07 (br, 6H). FT-IR $\left(\mathrm{KBr}, \mathrm{cm}^{-1}\right): 2927 \quad\left(v_{\mathrm{CH} 2}\right), 1781 \quad\left(\mathrm{v}_{\mathrm{asC}=\mathrm{O}}\right), 1726$ $\left(v_{\mathrm{SC}=0}\right), 1671 \quad\left(v_{\mathrm{C}=0}\right), 1527\left(v_{\mathrm{asNO} 2}\right), 1504\left(v_{\mathrm{C}=\mathrm{C}}\right)$, $1256\left(v_{\mathrm{C}-\mathrm{N}}\right) \cdot M_{\mathrm{n}}=1700, M_{\mathrm{w}} / M_{\mathrm{n}}=1.29$.

\subsection{Photochemical reaction of PSPIs}

PSPI films on quart, $\mathrm{KBr}$ or glass substrate were prepared for UV-vis, FT-IR and water contact angle measurement, respectively. The films were prepared by spin-coat of the PSPI solution in DMF. UV irradiation to the PSPI films was conducted by USHIO super high pressure mercury lamp Optical Module X with optical filters HA50 and UV-33 with the intensity of $8.8 \mathrm{~mJ} / \mathrm{cm}^{2}$ (at $365 \mathrm{~nm}$ ).

\section{Results and Discussion}

\subsection{Polymer Synthesis}

Photosensitive $o$-nitrobenzyl group-containing diamine monomer 1 was prepared chemoselective esterification of MBAA and 2.2 moleq. of $o$-nitrobenzyl chloride with 2.2 moleq. of DBU in DMSO. Small amount of the side products with $o$-nitrobenzyl amine moieties, which were formed by the reaction of amine and benzyl chloride, were carefully removed by column chromatography. FT-IR spectrum of diamine monomer 1 shows characteristic ester and nitro group peaks at 1689 and $1552 \mathrm{~cm}^{-1}$, respectively.

$o$-Nitrobenzyl group-containing PSPI 1-4 were prepared by polyaddition reaction of diamine monomer 1 with various carboxylic dianhydrides (PDA, 6-FDA, BTDA or PCDA) and subsequent chemical imidization in the presence of pyridine and acetic anhydride (Scheme 1). The resultant polymer was purified by reprecipitation into $\mathrm{MeOH} . \quad{ }^{1} \mathrm{H}-\mathrm{NMR}$ spectrum (Figure 1) showed benzyl methylene peak at 5.8-5.2 ppm and the peak intensity of the peak showed complete

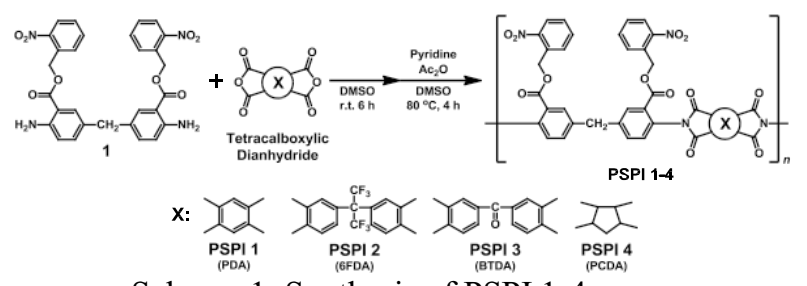

Scheme 1. Synthesis of PSPI 1-4

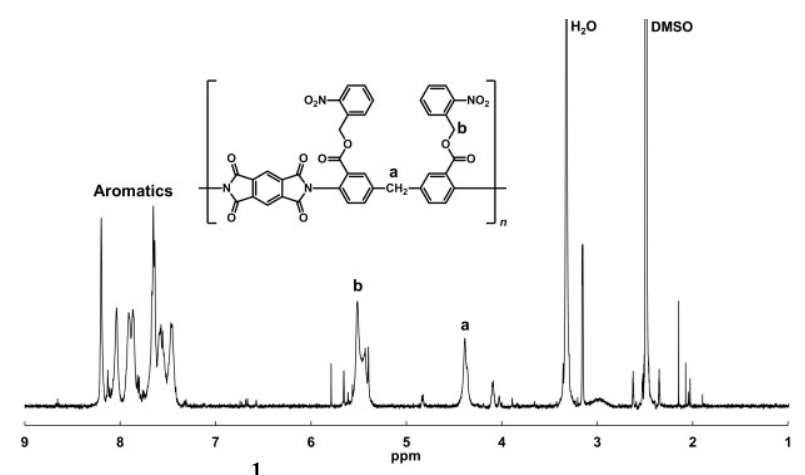

Figure 1. ${ }^{1}$ H-NMR spectrum of PSPI-1.

introduction of $o$-nitrobenzyl ester group in the polymer side chain. FT-IR spectra showed carbonyl peaks corresponding to imide group at around 1780 and $1725 \mathrm{~cm}^{-1}$. The molecular weight of the PSPIs was ranging from 12,000 to 1,700 which is relatively lower than that of conventional polyimides. Because the electron density of amine group in MBAA decreased by the electron-withdrawing carbonyl group at ortho-position.

PSPI 1-4 were soluble in THF, halogenic solvent $\left(\mathrm{CHCl}_{3}\right)$ and amide solvent (DMF, DMAc) and DMSO. PSPI 2 was soluble even in acetone, due to trifluoromethyl group in the polymer chain. Glass transition temperature ( $\left.T_{\mathrm{g}}\right)$ of PSPI 1-3 was around $170{ }^{\circ} \mathrm{C}$ and thermal decomposition temperature $\left(T_{\mathrm{d} 10}\right)$ was displayed over $300{ }^{\circ} \mathrm{C}$. In contrast, PSPI 4 displayed relatively lower $T_{\mathrm{g}}$ $\left(122{ }^{\circ} \mathrm{C}\right)$ and $T_{\mathrm{d} 10}\left(289{ }^{\circ} \mathrm{C}\right)$ because of the low molecular weight. 


\subsection{Photochemical reaction of PSPIs}

The FT-IR spectra of PSPI 4 films before and after UV irradiation were shown in Figure 2. The intensity of nitro group peak at $1530 \mathrm{~cm}^{-1}$ decreased. This data clearly indicated that the polarity change by conversion of $o$-nitrobenzyl ester to $o$-nitroso benzaldehyde and benzoic acid moiety in the polyimide chain was successfully conducted. The conversion of nitro group increased but gradually saturated by increasing of the UV irradiation time (Figure 3) After $90 \mathrm{~min}$ UV irradiation, the conversion of nitro group was reached to 46 (PSPI 2)-38\% (PSPI 4).

UV-vis spectra change of PSPI film on the UV irradiation was shown in Figure 4. The absorbance from 300 to $600 \mathrm{~nm}$ increased with the UV irradiation time which indicates the photochemical reaction of $o$-nitrobenzyl ester group was successfully occurred. Before UV irradiation, the transmittance of PSPI at $360 \mathrm{~nm}$ was ranging from 90 (PSPI 2) to 48\% (PSPI 3). The high transmittance of PSPI 2 is attributed to the lower charge transfer formation due to the trifluoromethyl group. The transmittance of all PSPI decreased by UV irradiation and saturated about $90 \mathrm{~min}$ by the shuttering effect. This shuttering effect should be caused by the removal of bulky $o$-nitrobenzyl ester group resulted in the enhancement of the charge transfer. Furthermore, the shuttering effect prevented the photochemical reaction for the conversion of $o$-nitrobenzyl

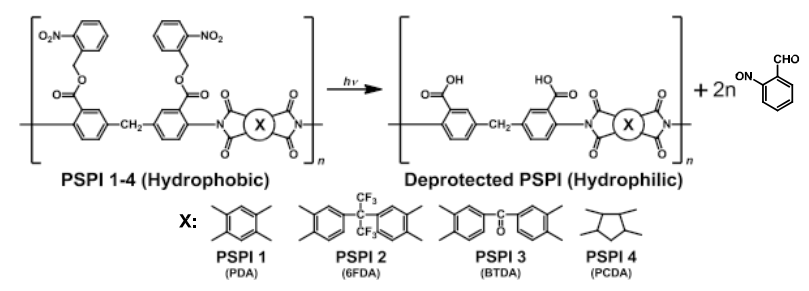

Scheme 2. Photochemical reaction of PSPI 1-4

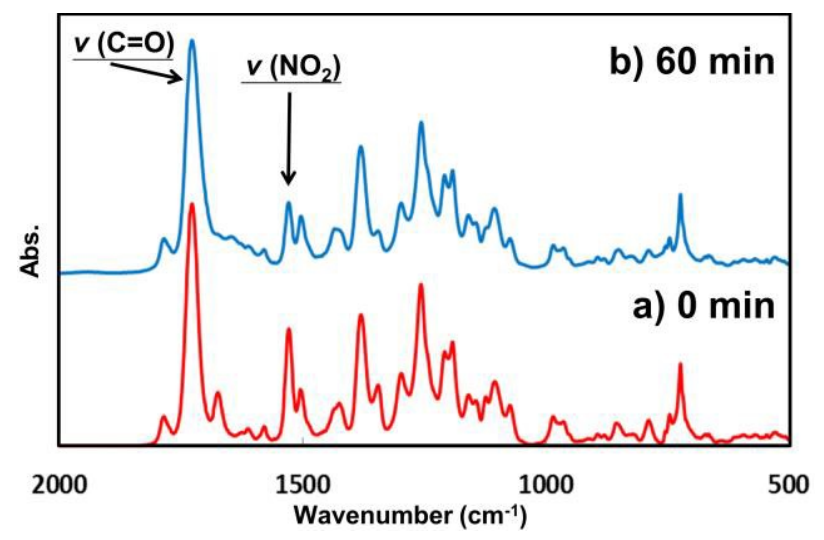

Figure 2. FT-IR spectra of PSPI 4. (a) before and (b) after UV irradiation. group as shown in Figure 2.

\subsection{Water Contact Angle of PSPIs}

PSPI 1-4 films was prepared on the glass substrate and the relationship between water contact angle and UV irradiation time was monitored (Figure 5). Before UV irradiation, all PSPI films showed water contact angle of around $75^{\circ}$. After UV irradiation for $60 \mathrm{~min}$, however, contact angle didn't show any change despite the photochemical reaction of nitro benzyl group to the generation of benzoic acid moiety proceeded. These results would be due to the o-nitroso benzaldehyde, which is byproduct of the

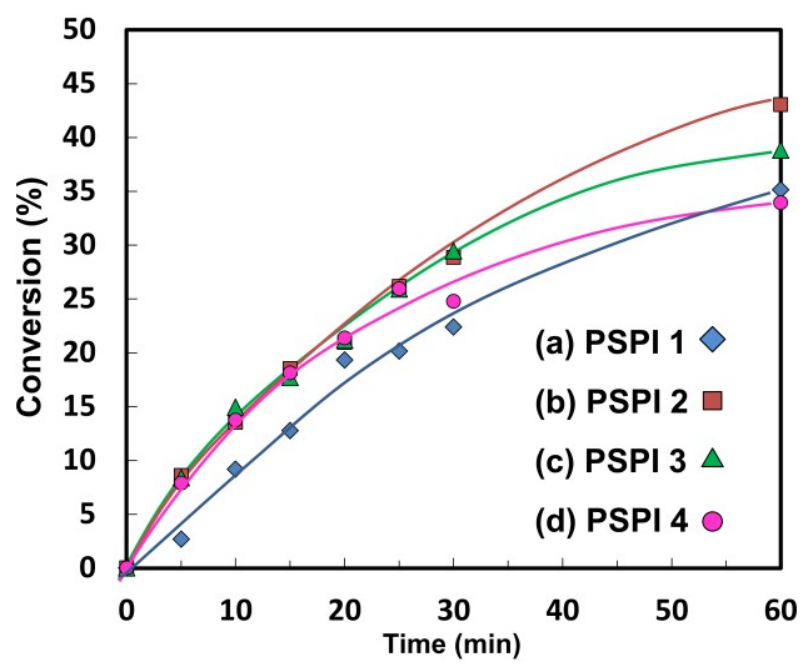

Figure 3. Relationship between irradiation time and $\mathrm{NO}_{2}$ group conversion of (a) PSPI 1, (b) PSPI 2, (c) PSPI 3, (d) PSPI 4 film.

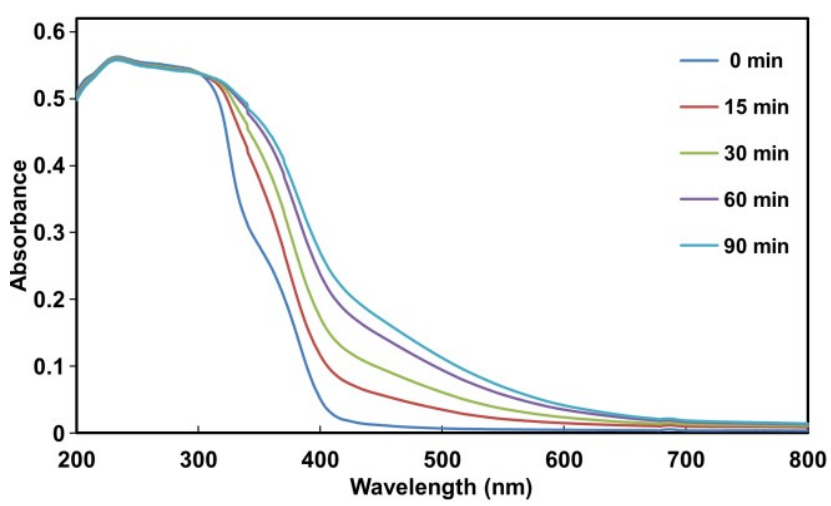

Figure 4. UV-vis spectra change of PSPI 3 film on UV irradiation.

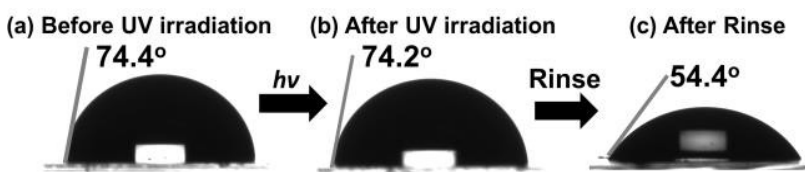

Figure 5. Water contact angle change of PSPI film (a) as cast, (b) after UV irradiation for $60 \mathrm{~min}$. (c) after rinse by toluene. 


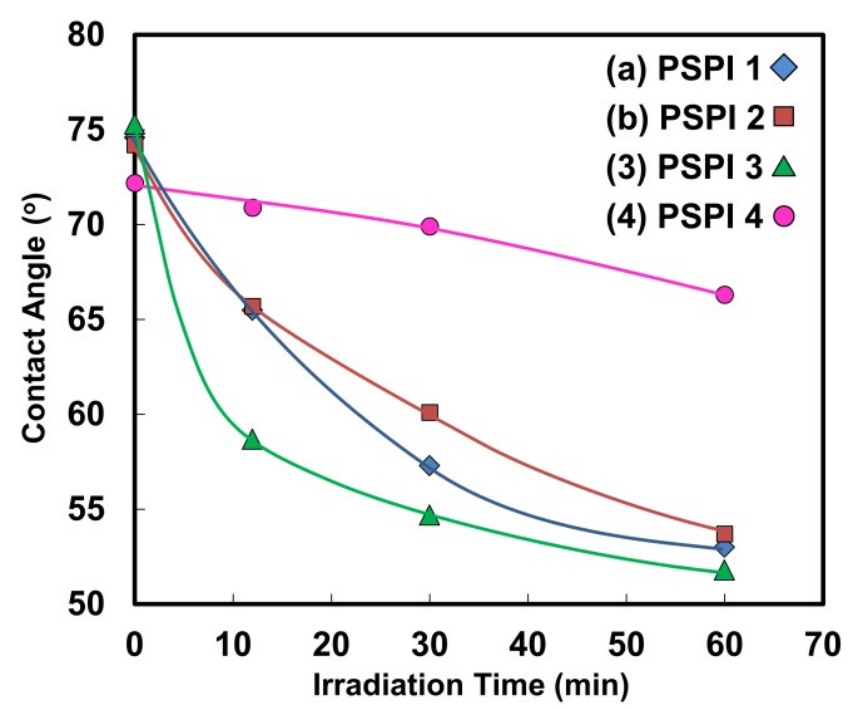

Figure 6. Relationship between irradiation time and water contact angle (a) PSPI 1, (b) PSPI 2, (c) PSPI 3, (d) PSPI 4 film.

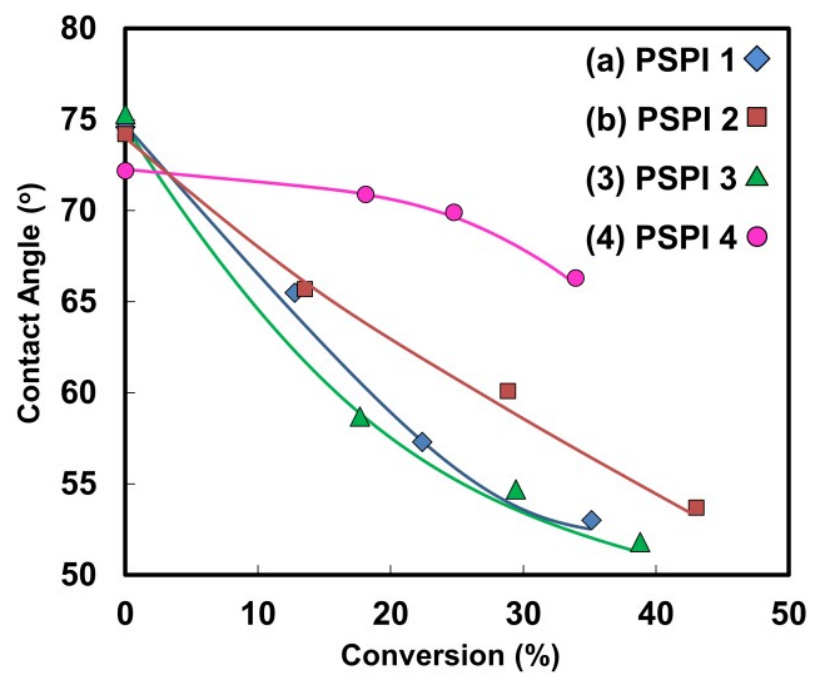

Figure 7. Relationship between conversion of $o$ nitrobenzyl group to water contact angle (a) PSPI 1, (b) PSPI 2, (c) PSPI 3, (d) PSPI 4 film.

photochemical reaction of $o$-nitrobenzyl ester, covered the PSPI film surface. To remove the $o$-nitroso benzaldehyde from polymer film, we demonstrated rinse of PSPI films by toluene after UV irradiation. Because toluene is good solvent for $o$-nitroso benzaldehyde, while poor solvent for PSPI 1-4. After rinse by toluene, the water contact angle decreased depending on the UV irradiation time was shown in Figure 6. Water contact angle of PSPI 1-3 decreased form $75^{\circ}$ to below $55^{\circ}$ in $60 \mathrm{~min}$ irradiation. These data suggest that the removal of $o$-nitroso benzaldehyde and the polar carboxylic acid group was emerged by toluene rinse. The water contact angle is linearly decreased by increasing the conversion of $o$-nitrobenzyl ester group which is corresponding to the density of polar benzoic acid group in the polymer film. Small change in the water contact angle of PSPI 4 is would be due to the hydrophobic nature of the aliphatic polymer main chain.

\section{Conclusion}

Photosensitive polyimides with pendant $o$-nitrobenzyl ester group were synthesized from the $o$-nitrobenzyl ester-containing diamine $\mathbf{1}$ and a series of tetracarboxylic dianhydrides. Diamine monomer 1 was prepared by chemoselective esterification, of carboxylic acid containing diamine MBAA. The obtained polymers possess photosensitive $o$-nitrobenzyl ester groups in the all side chain. Polar benzoic acid group was generated by the photochemical reaction of the $o$-nitrobenzyl ester group, and 38 to $46 \%$ of $o$-nitrobenzyl ester group was converted by UV irradiation for $90 \mathrm{~min}$. Polarity change in the polymer chains was resulted in the water contact angle change of PSPIs film. The water contact angle of PSPI films decreased from $75^{\circ}$ to $55^{\circ}$ by UV irradiation and subsequent rinse with toluene to remove $o$-nitroso benzaldehyde as the side product of the photochemical reaction.

\section{References}

1. M. K. Ghosh and K. L. Mittal, Polyimides: Fundamentals and Applications, Marcel Dekker, New York, 1996.

2. N. Yoda, Polym. Adv. Technol., 8 (1996) 215-226.

3. A. Mochizuki, T. Teranishi, M. Ueda, K. Matsushita, Polymer, 36 (1995) 2153-2158.

4. M. Ueda, T. Nakayama, Macromolecules, 29 (1996) 6427-3631

5. S. L.-C. Hsu, M. H. Fan, Polymer, 45 (2004) 1101-1109.

6. S. Ryu, J. H. Kim, S. H. Lee, M. H. Lee, Bull Korean Chem. Soc., 29 (2007) 1689-1694.

7. G. J. Shin, J. C. Jung, J H. Chi, T. H. OH, J. B.Kim, J. Polym. Sci. Part A: Polym. Chem., 45 (2007) 776-788

8. K. G. Choi, J. C. Jung, K. S. Kim, J. B. Kim,Polym, Adv, Technol., 16 (2005) 387-392.

9. A. Kameyama, T. Nishikubo, $J$. Photopolym.,Sci. Technol., 17 (2004), 45-50.

10. H. Tojo, A. Kameyama, T. Nishikubo, Chem.Lett., 27 (1998) 433-434.

11. H. Tojo, A. Kameyama, T. Nishikubo, $J$. Photopolym. Sci. Technol., 12 (1999) 223-230. 